\title{
123I-MIBG Imaging: Patient Preparation and Technologist's Role
}

\author{
S. Seth Van Vickle, CNMT, NCT, and Randall C. Thompson, MD, FASNC \\ Saint Luke's Mid America Heart Institute and Saint Luke's Cardiovascular Consultants, Kansas City, Missouri
}

CE credit: For CE credit, you can access the test for this article, as well as additional JNMT CE tests, online at https://www.snmmilearningcenter.org. Complete the test online no later than June 2018. Your online test will be scored immediately. You may make 3 attempts to pass the test and must answer $80 \%$ of the questions correctly to receive $1.0 \mathrm{CEH}$ (Continuing Education Hour) credit. SNMMI members will have their CEH credit added to their VOICE transcript automatically; nonmembers will be able to print out a CE certificate upon successfully completing the test. The online test is free to SNMMI members; nonmembers must pay $\$ 15.00$ by credit card when logging onto the website to take the test.

\begin{abstract}
The radiopharmaceutical ${ }^{123}$ I-metaiodobenzylguanidine (MIBG) was approved by the Food and Drug Administration in March 2013 for the assessment of myocardial sympathetic innervation in the evaluation of patients with heart failure and an ejection fraction of no more than $35 \%$. Almost any well-equipped nuclear medicine or nuclear cardiology laboratory can perform this test, although there is a need for special attention to patient preparation, dose calibration, and proper timing of the image acquisition. This article reviews the role of the nuclear medicine technologist and some practical aspects of cardiac sympathetic ${ }^{123}$ I-MIBG imaging of which the laboratory team needs to be mindful.
\end{abstract}

Key Words: ${ }^{123}$-MIBG; cardiac sympathetic imaging; cardiac denervation; techniques for MIBG imaging

J Nucl Med Technol 2015; 43:82-86

DOI: $10.2967 /$ jnmt.115.158394

$\mathbf{T}$ he radiopharmaceutical ${ }^{123}$ I-metaiodobenzylguanidine (MIBG) (AdreView; GE Healthcare) was approved by the Food and Drug Administration in March 2013 for the assessment of myocardial sympathetic innervation in patients with New York Heart Association class 2 or 3 heart failure and an ejection fraction of less than $35 \%$ (1). The role of this test in clinical practice is currently evolving. This article outlines the practicalities of the use of ${ }^{123}$ I-MIBG.

The use of ${ }^{123}$ I-MIBG can be best demonstrated by a case presentation. A 65-y-old man presents with mild limitations due to shortness of breath on exertion. He had a heart attack 2 y previously and has been somewhat limited ever since. A recent myocardial perfusion imaging test showed left ventricular scarring but no ischemia and a left ventricular ejection fraction of $36 \%$. However, a recent echocardiogram

\footnotetext{
Received Mar. 26, 2015; revision accepted Apr. 16, 2015.

For correspondence or reprints contact: S. Seth Van Vickle, Saint Luke's

Cardiovascular Consultants, 4330 Wornall Rd., Kansas City, MO 64111.

E-mail: svanvickle@saint-lukes.org

Published online May 8, 2015.

COPYRIGHT (C 2015 by the Society of Nuclear Medicine and Molecular Imaging, Inc.
}

obtained during a hospitalization at another facility showed an ejection fraction of $33 \%$. The patient is advised to consider an implantable cardioverter defibrillator but is reluctant because a relative has an implantable cardioverter defibrillator that frequently fires inappropriately.

It is certainly clear that implantable cardioverter defibrillators save lives; their prophylactic implantation in patients who have known heart failure with low ejection fractions is considered standard therapy (2). However, these devices are sometimes associated with complications and are expensive, and many are implanted for each life saved $(3,4)$.

For patients such as this one, who have borderline indications for implantable cardioverter defibrillators and are reluctant to proceed, physicians have a few options: accept that the patient does not want the device; try to convince the patient to have the device implanted; define the ejection fraction more exactly via another diagnostic medical imaging modality; or perform cardiac sympathetic imaging with ${ }^{123}$ IMIBG for risk stratification. The heart-to-mediastinum ratio provided by ${ }^{123}$ I-MIBG imaging is a powerful prognostic indicator in these patients, and such information may be useful in shared decision making by physicians and patients $(5-8)$.

\section{EQUIPMENT PREPARATION}

To properly acquire ${ }^{123} \mathrm{I}$-MIBG images, the camera must allow for frontal planar imaging. Anterior frontal plane images are a requirement, whereas SPECT images are optional but are usually obtained. The nuclear cardiology system should have low-energy, high-resolution collimation, and the camera should be able to obtain specific sequences from the imaging protocol, for example, static and SPECT imaging. Setting up and testing the imaging sequence before the day of dosing is often helpful and recommended.

The dose calibrator should also be inspected before the study is ordered. The dose calibrator should have a ${ }^{123} \mathrm{I}$-specific setting, and a correction factor specific for the laboratory's instrument must be obtained. It is strongly recommended that a commercial copper sleeve or copper tube be used during dose calibration to lessen scatter counts from low-energy photons, which can give falsely low readings for ${ }^{123}$ I-MIBG (9). 


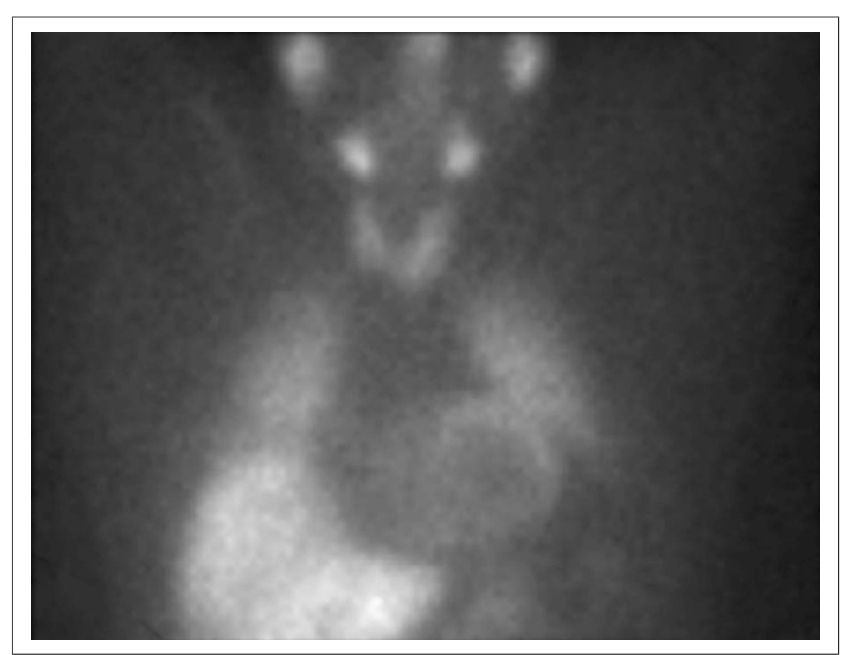

FIGURE 1. Anterior view of ${ }^{123}$ I-MIBG 3 h 50 min after injection.

Two static anterior planar acquisitions are obtained when imaging with ${ }^{123} \mathrm{I}-\mathrm{MIBG}$ (at $15 \mathrm{~min}$ and $3 \mathrm{~h} 50 \mathrm{~min}$ after injection). With a dual-head camera, the detectors should be positioned in the anterior and left lateral positions. The anterior field of view (FOV) will be focused on acquiring the myocardium and the mediastinal region for later processing (Fig. 1). The organs of interest should be positioned in the center of the field of view, with the apex of both lungs kept close to the top central field of view and the base of the myocardium kept in the bottom of the central field of view.

A 10 -min anterior acquisition in a $128 \times 128 \times 16$ matrix allows for adequate counting statistics. Photon acquisition should be centered on a $159-\mathrm{keV}$ peak with a $20 \%$ window.

\section{PATIENT PREPARATION}

When a clinical laboratory is preparing a patient for cardiac ${ }^{123}$ I-MIBG imaging, most of the steps typical of nuclear cardiology imaging are followed. The imaging team should be mindful of the patient's past medical history, including allergies, medications, and the general health history. There are also some medical issues particular to ${ }^{123}$ I-MIBG imaging. ${ }^{123}$ I-MIBG is contraindicated in patients with known hypersensitivity to iobenguane or iobenguane sulfate; however, it is also customary to screen potential patients for allergies to iodine-containing materials by asking if they have had any prior reactions to iodinated contrast material, shellfish, strawberries, or cranberries.

Particular attention must be paid to medication history. Table 1 provides a list of medications that affect ${ }^{123}$ I-MIBG imaging. This list includes medicines that the patient may not record unless specifically queried, such as over-the-counter cold medicines and cocaine. Certain common cardiac medications, including labetalol and calcium channel antagonists, also need to be stopped temporarily before ${ }^{123}$ I-MIBG imaging.

TABLE 1

Medications That Affect ${ }^{123}$-MIBG Imaging (13)

\begin{tabular}{|c|c|c|c|}
\hline Drug or class & Examples & $\begin{array}{l}\text { Mechanism of interference } \\
\text { (known or expected) }\end{array}$ & $\begin{array}{l}\text { Discontinuation before } \\
\text { 123/-MIBG scan (d) }\end{array}$ \\
\hline Opioid & & Uptake inhibition & $7-14$ \\
\hline Cocaine & & Uptake inhibition & $7-14$ \\
\hline Tramadol & & Uptake inhibition & $7-14$ \\
\hline $\begin{array}{l}\text { Tricyclic } \\
\text { antidepressants }\end{array}$ & $\begin{array}{l}\text { Amitriptyline and derivatives, imipramine } \\
\text { and derivatives, amoxapine, doxepin, } \\
\text { others }\end{array}$ & Uptake inhibition & $7-21$ \\
\hline Sympathicomimetics ${ }^{*}$ & $\begin{array}{l}\text { Phenylpropanolamine, ephedrine, } \\
\text { pseudoephedrine, phenylephrine, } \\
\text { amphetamine, dopamine, isoproterenol, } \\
\text { salbutamol, terbutaline, fenoterol, } \\
\text { xylometazoline }\end{array}$ & Depletion of granules & $7-14$ \\
\hline \multirow[t]{4}{*}{$\begin{array}{l}\text { Antihypertensive } \\
\text { or cardiovascular agents }\end{array}$} & Labetalol & $\begin{array}{l}\text { Inhibition of uptake } \\
\text { and depletion }\end{array}$ & 21 \\
\hline & Reserpine & $\begin{array}{l}\text { Depletion and } \\
\text { transport inhibition }\end{array}$ & 14 \\
\hline & Bretylium, guanethidine & $\begin{array}{l}\text { Depletion and } \\
\text { transport inhibition }\end{array}$ & 14 \\
\hline & $\begin{array}{l}\text { Calcium channel blockers (nifedipine, } \\
\text { nicardipine, amlodipine) }\end{array}$ & $\begin{array}{l}\text { Increased uptake and } \\
\text { retention }\end{array}$ & 14 \\
\hline \multirow[t]{4}{*}{ Antipsychotics } & $\begin{array}{l}\text { Phenothiazines }{ }^{\dagger} \text { (chlorpromazine, } \\
\text { promethazine, fluphenazine, others) }\end{array}$ & Uptake inhibition & $21-28$ \\
\hline & Thioxanthenes (maprotiline, trazodone) & Uptake inhibition & $21-28$ \\
\hline & Butyrophenones (droperidol, haloperidol) & Uptake inhibition & $21-28$ \\
\hline & Loxapine & Uptake inhibition & $7-21$ \\
\hline
\end{tabular}

*Components of bronchodilators, decongestants, and diet aids.

${ }^{\dagger}$ Frequent components of antiemetic and antiallergic agents. 


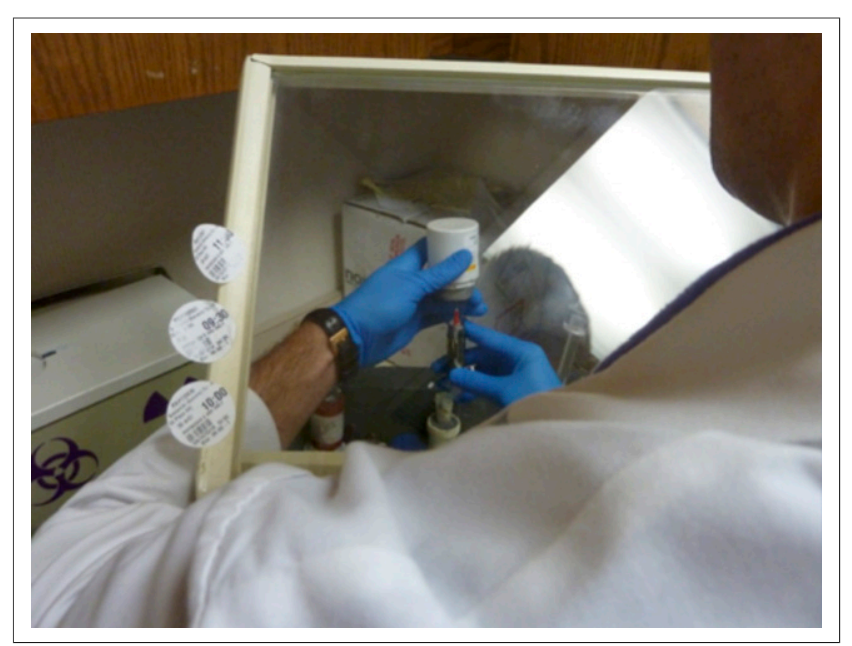

FIGURE 2. Proper technique for drawing a ${ }^{123}$ I-MIBG dose.

Two particularly relevant medical conditions include Parkinson disease and kidney disease. Patients with Parkinson disease have impaired cardiac uptake of ${ }^{123}$ I-MIBG (10). Renal insufficiency is considered a relative contraindication for ${ }^{123}$ I-MIBG testing since the radiopharmaceutical is cleared by the kidneys, although a recent report called into question this conventional position (11).

Patients who have an increased risk for thyroid cancer should be considered for potassium iodide pretreatment in order to block thyroid uptake and to lower the radiation dose to the thyroid $(1,12)$. Patients at increased risk would presumably include young patients and those with a personal or family history of thyroid cancer. Potassium iodine was not routinely administered before ${ }^{123}$ I-MIBG in the pivotal ADMIRE-HF trial (5).

Patients should be informed of key elements of the ${ }^{123} \mathrm{I}$ MIBG test beforehand to help alleviate their uncertainty and apprehension. Nuclear medicine tests can sound frightening to some people, so it is important to outline the procedure, time requirements, and reasons for the test. Patients are generally instructed to be well hydrated before the test, but care should be taken with heart failure patients who are on fluid restriction.

Radiation dosimetry should also be mentioned to the patient. The effective dose for an adult is $5.07 \mathrm{mSv}$ for the injection of $370 \mathrm{MBq}(10 \mathrm{mCi})$ of ${ }^{123} \mathrm{I}(13.7 \mu \mathrm{Sv} / \mathrm{MBq} \times$ $370 \mathrm{MBq}$, or $0.507 \mathrm{mSv} / \mathrm{mCi} \times 10 \mathrm{mCi})(1)$. There is also a small amount of radiation exposure from attenuation correction. The total exposure is relatively low compared with other procedures in nuclear cardiology and is comparable to about 15 mo of average background radiation.

The cost and logistics of this radiopharmaceutical cause the no-show policy to be more important than usual. ${ }^{123}$ I-MIBG is a particularly expensive radiopharmaceutical, and the dose travels a long distance before administration. Health insurance companies, including the Centers for Medicare and Medicaid Services, allow nuclear cardiology facilities to bill patients for no-shows, but the laboratory must have a written policy in place and it is good practice to have patients acknowledge the policy in writing.

\section{RADIOPHARMACEUTICAL PREPARATION}

The dose of ${ }^{123}$ I-MIBG is calibrated to be $370 \mathrm{MBq}$ $(10 \mathrm{mCi})$ at 12:00 PM on the day of administration. The dose arrives in a single-use vial containing $5 \mathrm{~mL}$ of ${ }^{123} \mathrm{I}-\mathrm{MIBG}(74 \mathrm{MBq} / \mathrm{mL}$, or $2 \mathrm{mCi} / \mathrm{mL})$. The radiopharmaceutical expires $36 \mathrm{~h}$ after the calibration time and should be stored at between $68^{\circ} \mathrm{F}$ and $77^{\circ} \mathrm{F}$, following hospital safety protocols.

Using aseptic technique and proper radiation safety, the technologist should draw $370 \mathrm{MBq}(10 \mathrm{mCi})$ into a shielded syringe (Fig. 2). The syringe should be placed into the dose calibrator to verify the proper number of megabecquerels (or millicuries) before injection. The dose can then be administered to the patient via a large peripheral vein over 1-2 min. The intravenous line should be flushed with saline before isotope injection to verify proper venous access and again after injection to remove any accumulated residual activity. During the timed administration, the patient should be continually monitored for any hypersensitivity or other reactions to the medication, such as itching, burning, or tingling sensations at the injection site. A postinjection assay is performed on the syringe to determine residual radioactivity and the total activity administered to the patient.

\section{THE 123|-MIBG ACQUISITION}

Because of the short injection-to-scan time, the technologist should ensure that the camera is unoccupied before administering the pharmaceutical. Preparing the acquisition setup before injection is also helpful.

Exact timing of the acquisition is important; a stopwatch should be used. An early 10-min anterior planar image is obtained starting at $15 \mathrm{~min}$ after injection, and a late 10-min anterior planar image is obtained starting at $3 \mathrm{~h} 50 \mathrm{~min}$ after injection. These combined images

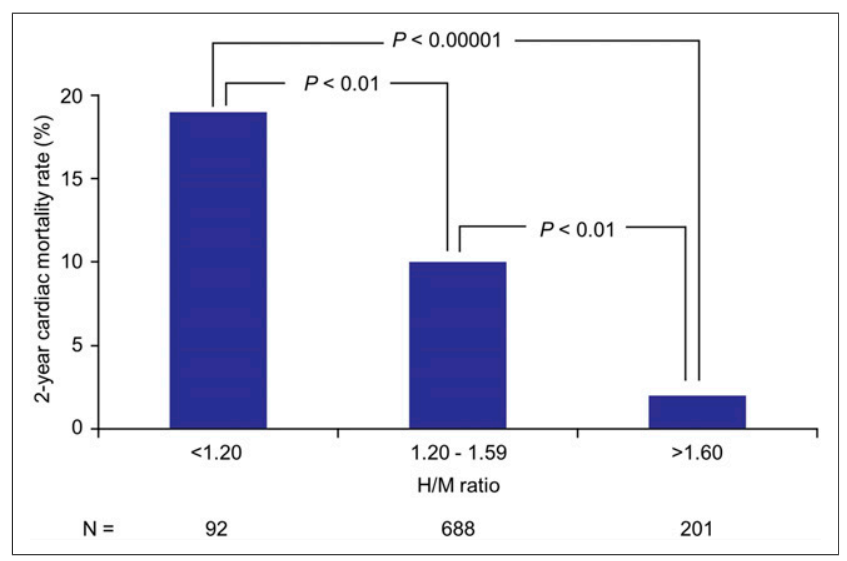

FIGURE 3. Association of cardiac death with heart-tomediastinum $(\mathrm{H} / \mathrm{M})$ ratio. 


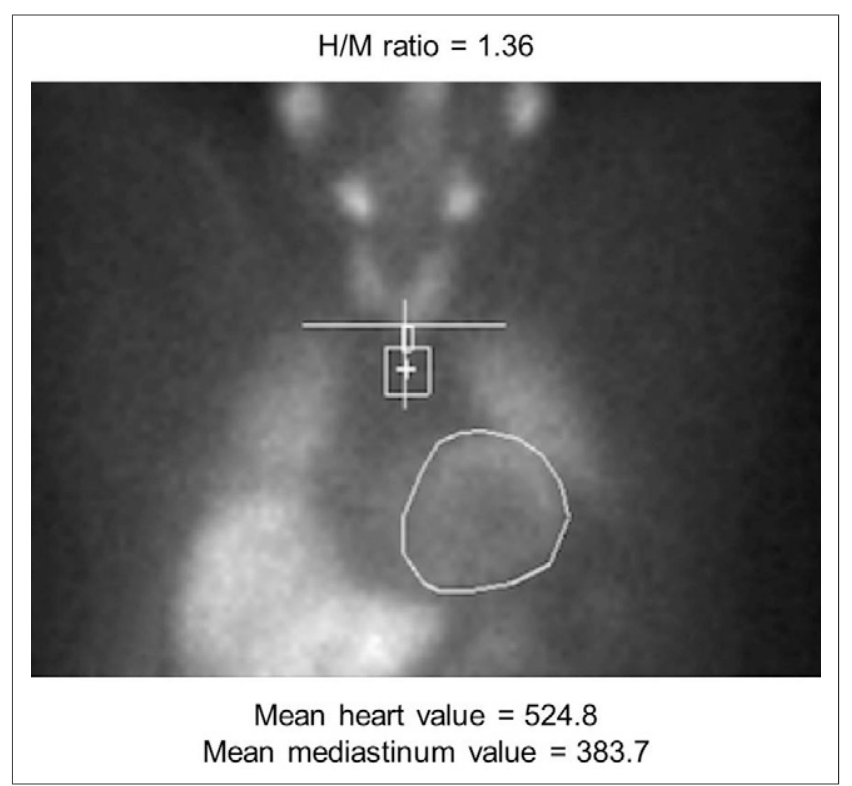

FIGURE 4. Heart-to-mediastinum (H/M) ratio obtained with 123|-MIBG.

allow for calculation of the washout ratio of the isotope (1).

Between these acquisitions, patients are allowed to eat and are encouraged to stay well hydrated but not excessively so if they have heart failure. Patients should also be advised that they are radioactive and to avoid small children and going through some airport security systems. SPECT images are acquired after the late planar acquisition.

The primary prognostic parameter supplied by ${ }^{123} \mathrm{I}-$ MIBG imaging is the heart-to-mediastinum ratio on the delayed images, which has been shown to predict the 2-y cardiac mortality rate (Fig. 3) (5). The regions of interest must be drawn around the heart and in the mediastinum in the prescribed fashion according to the product package insert. In brief, an irregular region of interest is drawn around the whole heart, defining the epicardial border. The number of counts and pixels in the region of interest are recorded. For the mediastinal region of interest, a horizontal line is drawn at the apices of the lungs. A vertical line is then drawn about equidistant between the right and left lungs. At the intersection of the horizontal and vertical lines, counts 15 pixels and below are marked. Starting with the fourth pixel below the intersection, the area with the lowest counts is identified and, if there are multiple areas, the most superior selected. At the lowest pixel, a $7 \times 7$ pixel region of interest is drawn, and the number of counts in this 49-pixel region of interest is recorded. The heart-tomediastinum ratio is equal to the counts per pixel in the cardiac region of interest divided by the counts per pixel in the mediastinal region of interest (Fig. 4).

\section{THE SPECT ACQUISITION}

The 4-h SPECT image dataset allows the clinician to visualize myocardial uptake and retention of ${ }^{123}$ I-MIBG in the classic short-, vertical-, and horizontal long-axis views. Our laboratory uses a circular orbit with $180^{\circ}$ of acquisition, and we acquire 64 projections using 32 stops of approximately $25 \mathrm{~s} /$ stop. An attenuation correction map is acquired simultaneously. The study is acquired in a $64 \times 64 \times 16$ matrix and is nongated.

\section{IMAGE PROCESSING}

Sample anterior planar images are shown in Figure 5. We customarily display the planar images in a 2-view panel (one image displaying the raw image and the other displaying the heart-to-mediastinum ratio along with the regions of interest).

The SPECT reconstruction parameters may vary depending on the site-specific routine, and the technologist should consistently use the reconstruction parameters best suiting the laboratory. The ${ }^{123}$ I-MIBG images are reconstructed in a manner similar to that of myocardial perfusion SPECT images. Either standard filtered backprojection or iterative reconstruction can be used. Typically, a low-pass filter is used, with a cutoff of 0.50 and an order of 5.00. The images are then displayed in a fashion similar to that of SPECT

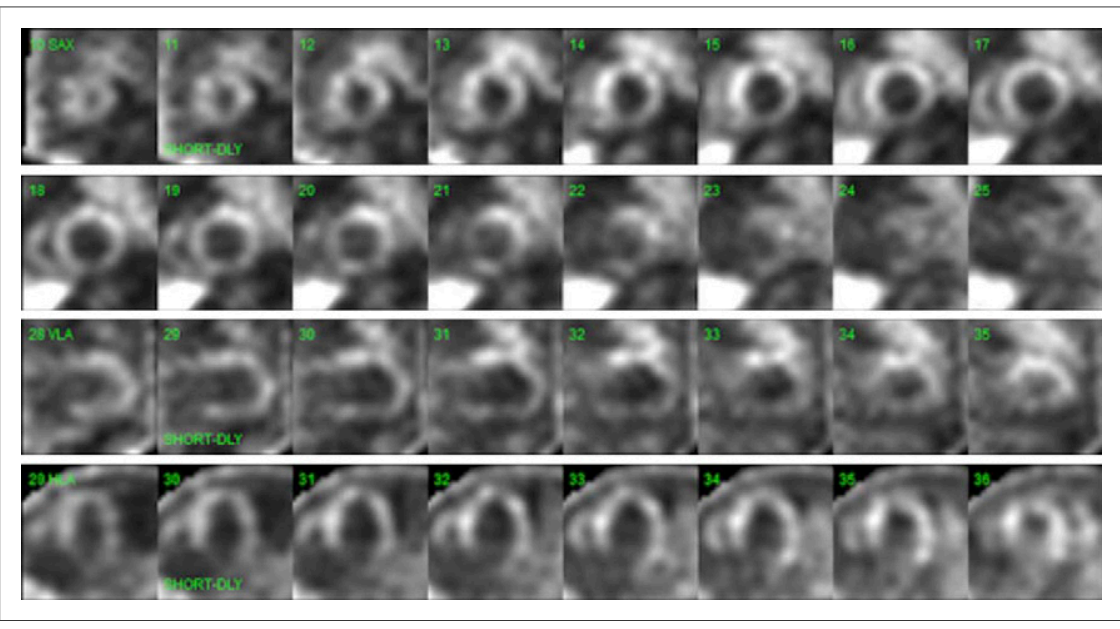

FIGURE 5. Classic SPECT myocardial display of ${ }^{123}$ I-MIBG images after reconstruction. 
myocardial perfusion images. Creating reconstruction defaults can help to standardize methods.

In 2010 Flotats et al. proposed standardization of ${ }^{123} \mathrm{I}$ MIBG cardiac sympathetic imaging on behalf of the European Association of Nuclear Medicine Cardiovascular Committee and the European Council of Nuclear Cardiology (13). Their publication provides more details about the acquisition and reporting of ${ }^{123}$ I-MIBG imaging.

\section{CONCLUSION}

Cardiac sympathetic ${ }^{123}$ I-MIBG imaging can be performed by almost all well-equipped nuclear medicine and nuclear cardiology laboratories. The procedure requires special attention to patient preparation, dose calibration, and timing of image acquisition. As opportunities to use this technique grow, its exact clinical role should become clear. The test is expected to provide clinicians with useful prognostic information about cardiovascular risk in patients with heart failure.

\section{DISCLOSURE}

No potential conflict of interest relevant to this article was reported.

\section{REFERENCES}

1. AdreView ${ }^{\mathrm{TM}}$ iobenguane I 123 injection. GE Healthcare website. http://www3. gehealthcare.com/en/products/categories/nuclear_imaging_agents/adreview. Published February 6, 2015. Accessed April 20, 2015.

2. Passman R, Goldberger JJ. Predicting the future: risk stratification for sudden cardiac death in patients with left ventricular dysfunction. Circulation. 2012;125: 3031-3037.
3. Vollmann D, Luthje L, Vonhof S, et al. Inappropriate therapy and fatal proarrhythmia by an implantable cardioverter-defibrillator. Heart Rhythm. 2005; 2:307-309.

4. Uslan DZ, Sohail MR, St Sauver JL, et al. Permanent pacemaker and implantable cardioverter defibrillator infection: a population-based study. Arch Intern Med. 2007;167:669-675.

5. Jacobson AF, Senior R, Cerqueira MD, et al. Myocardial iodine-123 meta-iodobenzylguanidine imaging and cardiac events in heart failure: results of the prospective ADMIRE-HF (AdreView Myocardial Imaging for Risk Evaluation in Heart Failure) study. J Am Coll Cardiol. 2010;55:2212-2221.

6. Tamaki S, Yamada T, Okuyama Y, et al. Cardiac iodine-123 metaiodobenzylguanidine imaging predicts sudden cardiac death independently of left ventricular ejection fraction in patients with chronic heart failure and left ventricular systolic dysfunction: results from a comparative study with signal-averaged electrocardiogram, heart rate variability, and QT dispersion. J Am Coll Cardiol. 2009;53:426-435.

7. Agostini D, Verberne HJ, Burchert W, et al. I-123-mIBG myocardial imaging for assessment of risk for a major cardiac event in heart failure patients: insights from a retrospective European multicenter study. Eur J Nucl Med Mol Imaging. 2008;35:535-546.

8. Nakata T, Nakajima K, Yamashina S, et al. A pooled analysis of multicenter cohort studies of ${ }^{123} \mathrm{I}$-mIBG imaging of sympathetic innervation for assessment of long-term prognosis in heart failure. JACC Cardiovasc Imaging. 2013;6:772-784.

9. Kowalsky RJ, Johnston RE. Dose calibrator assay of iodine-123 and indium-111 with a copper filter. J Nucl Med Technol. 1998;26:94-98.

10. Braune S, Reibhardt M, Schnitzer R, et al. Cardiac uptake of $\left({ }^{123} \mathrm{I}\right) \mathrm{MIBG}$ separates Parkinson's disease from multiple system atrophy. Neurology. 1999;53: 1020-1025.

11. Malhotra S, Wang L, Bunker $\mathrm{CH}$, et al. Renal dysfunction does not affect the prognostic value of myocardial iodine-123 meta-iodobenzylguanidine imaging in heart failure. Nucl Med Commun. 2014;35:58-63.

12. Friedman NC, Hassan A, Grady E, et al. Efficacy of thyroid blockade on thyroid radioiodine uptake in ${ }^{123}$ I-mIBG imaging. J Nucl Med. 2014;55: 211-215.

13. Flotats A, Carrió I, Agostini D, et al.; EANM Cardiovascular Committee; European Council of Nuclear Cardiology. Proposed standardization of ${ }^{123}$ I-metaiodobenzylguanidine (MIBG) cardiac sympathetic imaging by the EANM Cardiovascular Committee and the European Council of Nuclear Cardiology. Eur J Nucl Med Mol Imaging. 2010;37:1802-1812. 